\section{What's up doc? Reaching a diagnosis in the first-opinion consultation}

\section{Natalie Robinson', Rachel Dean', Malcolm Cobb' ${ }^{2}$, Marnie Brennan'}

1 Centre for Evidence-based Veterinary Medicine, The University of Nottingham, Nottingham, UK

2 School of Veterinary Medicine and Science, The University of Nottingham, Nottingham, UK

Understanding clinical decision-making during first-opinion veterinary consultations is vital to the progression of evidence-based veterinary medicine. Definitive diagnoses are not always reached, yet decisions on how to manage a case still need to be made, so it is currently unclear how making a diagnosis influences decision-making. The aim of this study was to determine how frequently a definitive diagnosis is made, the factors which influence this, such as the type of problem, the type of patient (e.g. species), which consulting veterinary surgeon is seen and which practice is visited, as well as the common diagnoses made.

Data were gathered during direct observation of firstopinion consultations in eight veterinary practices. Data were collected on all problems discussed during the consultation, including the type of diagnosis made. Diagnoses were categorised as: Definitive, Working, Presumed, Open or Previous, with definitions developed to ensure consistent coding. The specific diagnosis made, e.g. atopic dermatitis, was also recorded. A multi-level model was built to explore the influence of the problem and patient characteristics, as well as consulting veterinary surgeon and practice, on making a definitive diagnosis.

Data on diagnosis were gathered for 3192 health problems discussed for 1901 patients presented.The most common type of diagnosis made was Previous $(n=1116 / 3192$; $35.0 \%$ of all health problems), followed by Open ( $n=868 / 3192$; $27.2 \%)$ then Definitive $(n=660 / 3192 ; 20.7 \%)$. Presumed diagnoses were made for $15.0 \%(n=478 / 3192)$ of all problems discussed, while Working diagnoses were made for $2.2 \%$ $(n=70 / 3192)$. Characteristics of the individual problem, for example body system affected, had more influence over whether a definitive diagnosis was made than characteristics of the patient, veterinary surgeon or practice. Overweight/obese was the most common diagnosis made overall, followed by periodontal disease.

The common diagnoses identified can be used to prioritise topics for future research and guide veterinary curricula. However, given that definitive diagnoses are reached relatively infrequently during the consultation, it may be useful to focus future research and education around common clinical presentations, e.g. vomiting, rather than around a specific diagnosis. Further work is needed to understand how making a diagnosis influences the way in which health problems are managed, as well as how this influences long-term patient outcomes.

\section{Pedigree dog showing in the UK: Show exhibitors attitudes towards Pedigree dog health}

\section{Victoria Woods, Rachel Dean, Christina Kuhl, Richard Lea}

University of Nottingham, Nottingham, UK

The breeding of pedigree dogs to the relevant breed standard required for dog showing has raised health and welfare concerns. Since show exhibitors represent one influential stakeholder in this process they play a key role in pedigree dog health. The aim of this study was to gather opinions of UK exhibitors on current pedigree dog health breeding practices including its regulation by the Kennel Club and identify barriers to implementing health promotion strategies.

Data were collected by online survey and distributed using convenience sampling. Both quantitative and qualitative questions were included and 2090 exhibitors responded. Analysis was performed using basic descriptive statistics using SPSS and Excel data re-presented as whole numbers and \%.
Data analysis of 1866 respondents included 221 who exhibited a High Profile Breed (HPB) with the remainder of the respondents breeding and exhibiting, or just exhibiting Non-HPBs. The majority of surveyed exhibitors health test their breeding stock where recommended 97\% (1104/1139) and $90 \%(905 / 1005)$ think the results of these tests should be publically available. The majority of exhibitors believe an emphasis should be placed on health in the show ring 95\% (1376/1448).

When responding to questions regarding support received from the Kennel Club on their breeds' health, there were two substantial extreme groups; those who felt they received positive support $3.7 \%$ (55/1493), and those who felt they received none $10.9 \%(162 / 1493)$.

This lack of unity between breeders and the Kennel Club may be a factor which limits the improvement in pedigree dog health.

Exhibitors want to improve the health of their pedigree dogs. In order for them to succeed they must be supported by their governing body, there must be cooperation, communication, and consultation from all stakeholders regarding schemes and steps being taken to improve pedigree dog health. 